\title{
The resolution of Californian populations of Liriomyza huidobrensis and Liriomyza trifolii (Diptera: Agromyzidae) using PCR
}

\author{
D. J. W. MORGAN $\dagger$, S. R. REITZ $\ddagger$ P. W. ATKINSON $\dagger \&$ J. T. TRUMBLE* \\ $\dagger$ Department of Entomology, University of California, Riverside, CA 92521, $\vdots$ USDA-ARS, Center for Biological \\ Control, 310 South Perry-Paige Bldg. Florida A \& M University, Tallahassee, FL 32307, U.S.A
}

\begin{abstract}
Liriomyza trifolii (Burgess) and Liriomyza huidobrensis (Blanchard) are important vegetable pests in California. Populations of each species differ in their impact in central and southern regions. This difference may be explained by geographical or host plant differences in each of the regions. We used random amplified polymorphic DNA polymerase chain reactions to assess genetic differences between two laboratory populations of each species collected from central and southern California. Individual L. trifolii from the two regions could be discriminated by the presence/absence of PCR products. No such qualitative differences were apparent in PCR products amplified from L. huidobrensis individuals, but the origins of individuals could be differentiated using a bootstrap analysis of marker frequencies. Marker primers were used to compare field and laboratory individuals. No evidence was found for the existence of further populations or of hybrid populations in central and southern California. The distribution of populations of L. huidobrensis was explained completely by geographical differences. As a consequence of the absence of leafminer infestations on the same host plant varieties in both regions, factors governing L. trifolii population distribution differences were less apparent. The presence of the same host plant varieties at both sites suggests that the two L. trifolii populations differ in host plant preference.
\end{abstract}

Keywords: genetic marker, leafminer, Liriomyza huidobrensis, Liriomyza trifolii, RAPD-PCR.

\section{Introduction}

The agromyzid leafminers Liriomyza huidobrensis (Blanchard) and Liriomyza trifolii (Burgess) are distributed globally. Both species have spread throughout the world, becoming pests wherever vegetable and ornamental crops are cultivated (Spencer, 1973; Reitz et al., 1999). Their success as herbivores can be attributed largely to extreme polyphagy and the ability to rapidly develop resistance to pesticides. Both leafminer species are important pests of tomatoes, onions, bell peppers, celery, lettuce, and chrysanthemums (Spencer, 1973).

A number of observations suggest the existence of genetically different populations of $L$. huidobrensis and L. trifolii. First, the global distribution of these insects indicates adaptation of populations to localized conditions that range from holarctic to tropical (Spencer, 1973). Secondly, allopatric effects may result in differences in host plant preference between populations. For

\footnotetext{
*Correspondence. E-mail: john.trumble@ucr.edu
}

instance, L. huidobrensis is a major pest of tomatoes in Israel, but is rarely seen on this plant in California (Lange et al., 1957). Alternatively, allopatric effects may lead to changes in life histories; the optimum development temperature for L. trifolii on Phaseolus spp. is $32.5^{\circ} \mathrm{C}$ with a population from Florida, but is $35^{\circ} \mathrm{C}$ with a population from China (Charlton \& Allen, 1981; Chien \& Ku, 1996).

Evidence for genotypic variation within a polyphagous leafminer species has also been found in sympatric populations of Liriomyza sativae Blanchard. Host plant specialization of populations within the species was shown by back-crossing insects collected from tomatoes and cowpeas in North Carolina (Via, 1984a,b). However, no evidence was found for host race formation.

In this study we assessed the genetic variability of L. trifolii and L. huidobrensis populations from central and southern California using random amplified polymorphic DNA polymerase chain reactions (RAPDPCR). This technique has been used for a number of population biology and ecological studies (Arnheim et al., 1990; Hoy, 1994). The benefits of this technique 
include speed in identifying genetic polymorphisms, versatility in use for paternity, population, and species studies, and low cost (Williams et al., 1990). RAPDPCR is particularly useful in studies where little genetic information is available (Hoy, 1994).

Criticisms regarding the use of RAPD-PCR fall primarily into two groups; (i) the repeatability of the technique, and (ii) the difficulty in differentiating between homozygous and heterozygous genotypes (Haymer \& McInnis, 1993; Haymer et al., 1997). The former limitation can be minimized through careful control of conditions including DNA isolation, concentration, and amplification (Hoy, 1994). The latter criticism is relevant only in cases where results are expected to fit a particular frequency distribution for quantification of genetic distances and inheritance patterns, and when a low number of individuals or primers are used for evaluating genetic variability. We addressed this concern by designing a bootstrap program to resample data. This technique uses results from the RAPD-PCR reactions to build up a frequency distribution of similarities. Bootstrap resampling assumes that the sampled data provide the best available representation of the frequency distribution of the population as a whole (Crowley, 1992). A large sample size, either in terms of number of primers or number of individuals, is necessary to optimize the reliability of this assumption.

The first part of the study evaluated similarities of leafminer individuals within and between laboratory populations of leafminers initiated from field collections in central and southern California. Primers that differentiated between groups were subjected to further screening to assess their potential for identifying individuals from each population.

The second part of the study involved using these marker primers to test similarities of field collected individuals to laboratory populations. Three questions were addressed in the second part of the study: (i) Are the primers used for the discrimination of laboratory populations effective for identification of field populations?; (ii) Do field populations of leafminers segregate accord- ing to host plant or geographical region?; and (iii) Is there any evidence for population movement or mixing?

\section{Methods}

\section{Leafminer populations}

A minimum of 100 Liriomyza huidobrensis and Liriomyza trifolii, collected from central and southern California, were used to start four laboratory colonies of leafminers at the University of California, Riverside (Table 1). Several times after colony initiation, further individuals collected in the field were added to colonies to reduce risk of inbreeding. The insects were maintained on a variety of host plants according to availability.

\section{DNA isolation and amplification}

Each insect was macerated in 5\% (w/v) Chelex chelating reagent (Bio-Rad Laboratories, Richmond, CA). Solutions were incubated overnight $\left(56^{\circ} \mathrm{C}\right)$, then boiled for five minutes. The solution was diluted $10 \times$ for use in PCR reactions.

PCR reactions were set up using methodologies of PCR reactions used 4\% DNA template per reaction, $10 \% 10 \times$ assay buffer (Promega), 4\% mixed dNTPs $(2.5 \mathrm{M})$, and $0.4 \% \mathrm{Taq}$ polymerase ( 0.5 units). The PCR programmer protocol was: (a) $94^{\circ} \mathrm{C}$ for $2 \mathrm{~min}$; (b) $92^{\circ} \mathrm{C}$ for $2 \mathrm{~min}$; (c) $35^{\circ} \mathrm{C}$ for $2 \mathrm{~min}$; (d) ramp at $12^{\circ} \mathrm{C} \mathrm{min}^{-1}$ up to $72^{\circ} \mathrm{C}$; (e) $72^{\circ} \mathrm{C}$ for $2 \mathrm{~min}$; (f) cycle b-e 40 times; (g) $72^{\circ} \mathrm{C}$ for $5 \mathrm{~min}$; (h) hold at $4^{\circ} \mathrm{C}$. Each product was fractionated on 1.5\% TBE-agarose gel. Each run included $1 \mathrm{~kb}$ ladders, a PCR reaction without insect DNA as negative control, and PCR reactions from previously tested individuals of known origin as positive controls.

Gels were visualized with ethidium bromide staining and an image of the gel was captured using a UV light cabinet and image grabber. Bands were matched according to their size under the assumption that comigrating bands represented identical products.

Table 1 Location, species, and host plants from which leafminers were collected for initiation of colonies

\begin{tabular}{lcll}
\hline Species & Laboratory colony & \multicolumn{1}{c}{ Host Origin } & \multicolumn{1}{c}{ Plant } \\
\hline Liriomyza huidobrensis & Central & Monterey County & Lettuce \\
& Southern & San Diego County & Gypsophila \\
Liriomyza trifolii & Central & San Benito County & Bell Pepper \\
& Southern & Orange County & Celery \\
\hline
\end{tabular}


A preliminary PCR reaction was used to assess the quantity and quality of DNA extracts for PCR. The primer OPA08 (Operon Technologies, Alameda, CA) was used because it consistently gave a single band of similar intensity and size for each species. Only samples that produced the expected single product were used for future analyses. DNA Samples were diluted to approximately $10 \mathrm{ng} / \mathrm{l}$ using a calibration curve.

Banding patterns produced by primers were compared between individuals using a simple matching procedure (Nei \& Li, 1979). Pairwise similarity indices were calculated using the equation:

$F=2 N_{x y} /\left(N_{x}+N_{y}\right)$

where $N_{x}$ and $N_{y}$ are the number of RAPD = generated DNA fragments of differing sizes (bands) present in individuals $x$ and $y$, respectively; $N_{x y}$ is the number of bands common to both $x$ and $y$. The similarity index, $F$, varies from 0 to 1 for dissimilar to identical genotypes, respectively.

A bootstrap program was designed to assess band similarity between each individual and a randomly selected sample made up from individuals (a) within the same population, (b) between populations of the same species, and (c) between species. The randomization procedure was iterated 1000 times to assess mean similarities and 95\% confidence intervals for each comparison.

\section{Laboratory population studies}

Population similarity studies. Four individual leafminers ( 2 male, 2 female) from each of the four laboratory colonies were used to screen a total of 60 primers (sets OPA, OPB, and OPC, Operon Technologies, Alameda, CA). All products were scored according to their presence/absence for each individual. The complete dataset was used to assess within-population, betweenpopulation, and between-species similarities.

Identification of genetic markers. Primers were classified according to the variability of the PCR products that they produced between individuals. Primers that were identified as candidates for population markers within species were re-assayed using a further 10 individuals ( 5 male, 5 female) from each population to ensure consistent results were obtained with a larger sample size. PCR products that were consistent within populations, but differed between populations were used as markers to differentiate populations (Lynch \& Milligan, 1994).

We used two methods to identify the group origin of individuals using PCR markers. The qualitative approach involved products that are present in $100 \%$ of one population and absent in $100 \%$ of the other. Such markers are only likely to occur in populations that are reproductively isolated. The quantitative approach can be used when reproductive isolation is incomplete or when no qualitative markers can be found. Several primers, which identify products that differ in frequency between populations, can be used to estimate the probability of an individual belonging to a particular group. The degree of resolution depends on the number of products identified and their frequency in each population.

\section{Field population studies}

Plant foliage containing leafminer pupae were collected in central and southern California throughout 1997 and 1998. We recorded the location, date, and host plant from which leafminers were collected. Foliage with larvae was placed in containers and pupae isolated on emergence from the plant. Adult leafminers were stored at $-65^{\circ} \mathrm{C}$ until analysed.

We evaluated population identity using eight leafminers per population where possible. Populations were identified to species by visual inspection, with representative samples identified by E. Fisher (CDFA). Population identity was ascertained using appropriate marker primers from the laboratory population studies.

We calculated the similarity of field-collected individuals to laboratory populations using the bootstrap program described above. In order to associate an individual with a particular population it had to conform to three expectations: it must have a similarity index (i) in the $95 \%$ confidence limits of the withinpopulation similarities for one laboratory population; (ii) beyond the $95 \%$ confidence limits of the withinpopulation similarities for the other laboratory population; and (iii) in the $95 \%$ confidence limits of the between-population similarities when compared to that other laboratory population.

Are the primers used for the discrimination of laboratory populations effective for identification of field populations? All leafminers collected from the field were screened using the specific and appropriate markers, and classified according to similarities to each of the laboratory populations. Population similarities conforming to either population may indicate intermixing between populations or the existence of populations other than the two identified in the laboratory studies.

Insects with identity dissimilar from central and southern laboratory populations were investigated according to their origin, date of capture and host plant. Significant differences in unidentified individual 
frequencies at given locations, host plants, or dates, would indicate the existence of populations of leafminers in California other than those previously identified.

Do field populations of L. huidobrensis segregate according to host plant? Patterns in leafminer population distribution were investigated using analysis of variance (ANOVA). Generalized Linear Modelling (GLIM, Royal Statistical Society, London) allowed analysis of data conforming to a binomial distribution, and accounted for nonorthogonal sample size by weighting for denominator size. The dependent variable was the proportion of central individuals in the population. Only individuals that had been identified as belonging to either central or southern populations were included in the denominator. Host plant (7 species), collection date (year), leafminer species (2 species), and geographical origin (central and southern) were included as main effects and interactions in the analysis.

Is there any evidence for population movement or mixing? Liriomyza huidobrensis individuals were collected from celery host plants in Monterey (central) and Ventura (southern) counties, CA in 1997 and 1998. The population composition of each collection was identified using PCR protocols outlined above. The population composition at each locality was analysed using ANOVA to assess changes in population composition over time.

\section{Results}

\section{Laboratory population studies}

Population similarity studies. Within-population similarities were high for both species, ranging from 0.71 to 0.80 with no outlying individuals observed in any of the populations (Fig. 1a-d). The similarity between L. huidobrensis populations was not significantly different from the mean within-population similarity $(0.72 \pm$ 0.07 vs. $0.74 \pm 0.07$ mean $\pm 99 \%$ CI, respectively; Fig. 1e). In contrast, the between-population similarity for L. trifolii was significantly less than the mean withinpopulation similarity $(0.49 \pm 0.11$ vs. $0.76 \pm 0.12$ mean $\pm 99 \% \quad C I$, respectively), indicating distinct population differences at the genetic level (Fig. 1f). The similarity between species was significantly lower than any within-species comparisons $(0.19 \pm 0.10$, mean $\pm 99 \%$ CI; Figs 1e, f).

Identification of genetic markers. Of the 60 primers used, 29 could be used to differentiate between leafminer species, 16 could separate central and southern L. trifolii populations, but only 7 could separate between central and southern L. huidobrensis populations. A total of 16 primers was further tested with the larger sample size. For between-species differences, the four primers used were OPA05, 7, 10, and OPC03. Primers used for differentiating between populations of $L$. huidobrensis were OPA09, OPB14, OPC02, 5, 12, and 16, and between populations of $L$. trifolii were OPA02, 11, OPB01, 7, 15 , and OPC18.

All primers used for the differentiation between species were clear and differentiated consistently between species for all individuals tested (a total of 56 individuals). We selected OPA05 and 10 as marker primers for future studies because each primer produced a single distinct product consistent in size for all individuals of a species, but differed in size between species. Similarly, primers used to differentiate between populations of $L$. trifolii were clear and consistent over all individuals tested, however, OPB07 was not always clear, so was excluded from future studies.

For L. huidobrensis, only one primer (OPC05) consistently differentiated between central and southern populations. Three primers were capable of differentiating between populations in $81 \%, 86 \%$, and $96 \%$ of tests (OPA09, OPC02, and OPB14, respectively). The probability of observing these outcomes was not significantly different from an outcome of $100 \%$ resolution (Fisher's exact test, $P>0.11$ ). The remaining two tested primers were considered unreliable for differentiating between populations $(<70 \%$ efficient). Consequently, the four most reliable primers were selected for future studies, the use of several primers reduces the probability that differences in frequency distributions between laboratory and field colonies are an artifact of colony inbreeding.

The bootstrap similarity program estimated a probability of 0.983 for differentiating an individual L. huidobrensis belonging to the central population from southern population individuals, and 0.996 for identifying an individual $L$. huidobrensis belonging to the southern population from central population individuals. The difference in resolution between the two populations was due to the greater variability in band production between individuals within the central population (Fig. 2).

\section{Field population studies}

Are the primers used for the discrimination of laboratory populations effective for identification of field populations? All but one individual $L$. trifolii collected from the field $(n=65)$ were identified as similar to central or southern laboratory populations. We found no evidence for intermixing of populations in the field, nor for the existence of populations other than those identified from laboratory studies. 

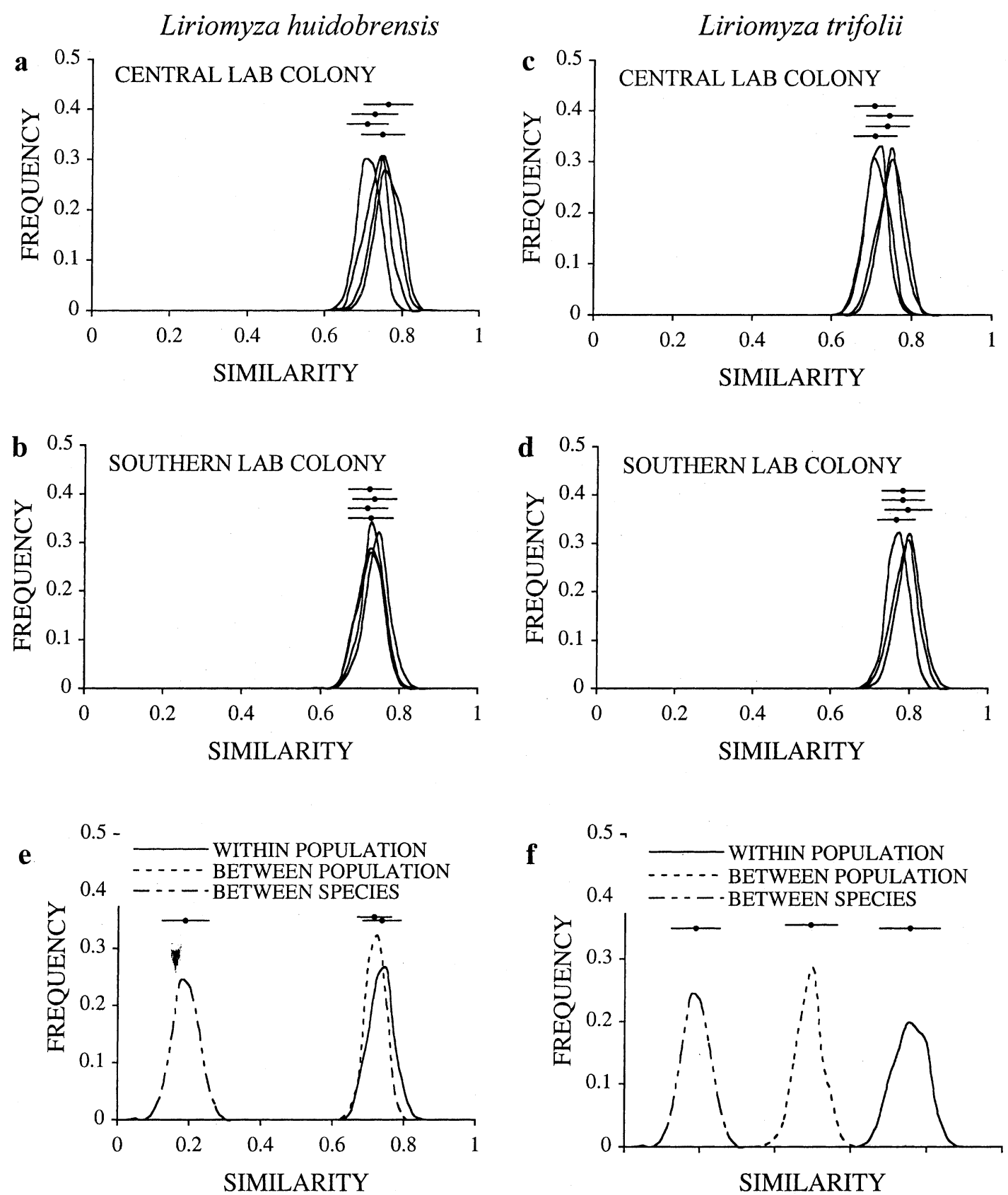

Fig. 1 RAPD-PCR derived similarity patterns for laboratory populations of L. huidobrensis (left) and L. trifolii (right). Bootstrapped distributions of comparisons between each individual within populations $(\mathrm{a}-\mathrm{d})$, between populations, and between species (e-f). Data points with horizontal error bars indicate mean similarities and $95 \%$ confidence intervals for each bootstrapped comparison.

The PCR markers from 5 field-collected L. huidobrensis individuals out of 110 did not conform to central or southern laboratory population marker frequencies, nor were the marker frequencies of the unidentified individuals similar to each other. If these were further L. huidobrensis populations than the two identified in this study, they would be expected to be grouped spatially, temporally, or according to host plant. The distribution of these individuals in the field was unrelated to the location $\left(F_{1,4}=1.120, P=0.34\right)$, date of capture $\left(F_{1,4}=1.323, \quad P=0.31\right)$, or host plant $\left(F_{8,4}=3.579, P=0.12\right)$. It is therefore unlikely that the five unidentified individuals belong to additional populations; it is likely instead that poor amplification 


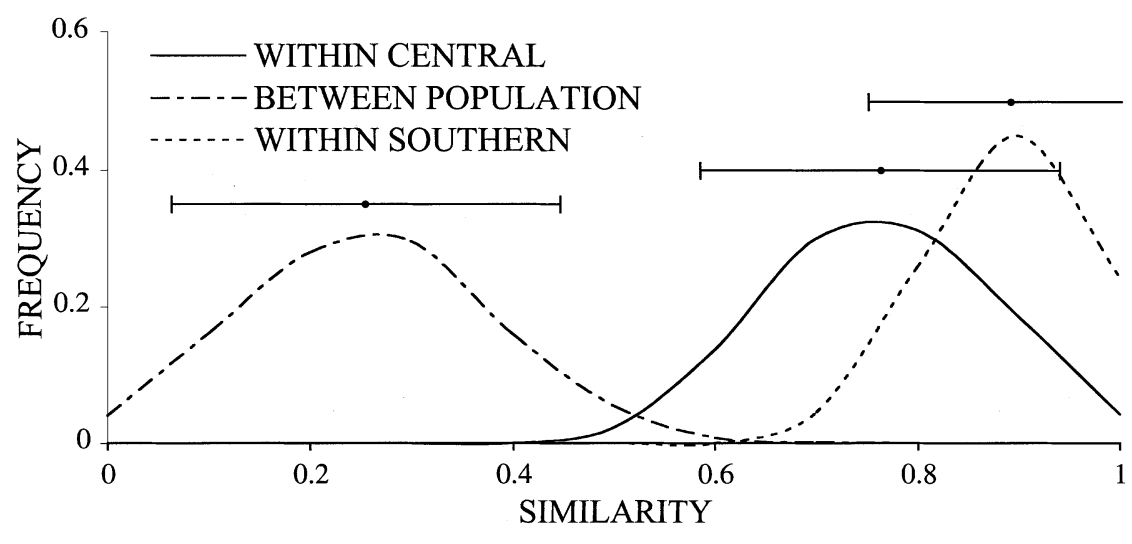

Fig. 2 PCR derived similarity patterns for laboratory populations of L. huidobrensis using marker primers. Data points with horizontal error bars indicate mean similarities and $95 \%$ confidence intervals for each bootstrapped comparison.

Table 2 Population identity of L. huidobrensis and L. trifolii individuals collected from various host plants at locations throughout central (C) and southern (S) California

\begin{tabular}{|c|c|c|c|c|c|c|}
\hline \multirow[b]{2}{*}{ Leafminer species } & \multicolumn{2}{|c|}{ Collection } & \multirow[b]{2}{*}{ Host plant } & \multicolumn{2}{|c|}{ Identified individuals } & \multirow[b]{2}{*}{ Unidentified individual } \\
\hline & Location & Date & & Central & Southern & \\
\hline \multirow[t]{10}{*}{ L. huidobrensis } & C (Monterey) & 1997 & Broccoli & 8 & 0 & 0 \\
\hline & C (Monterey) & 1997 & Cauliflower & 8 & 0 & 0 \\
\hline & C (Monterey) & 1997 & Chard & 7 & 0 & 1 \\
\hline & C (Monterey) & 1997 & Lettuce & 8 & 0 & 0 \\
\hline & C (Monterey) & 1997 & Lettuce & 8 & 0 & 0 \\
\hline & C (San Benito) & 1997 & Onions & 8 & 0 & 0 \\
\hline & C (Monterey) & 1997 & Spinach & 7 & 0 & 1 \\
\hline & C (Monterey) & 1998 & Romaine & 8 & 0 & 0 \\
\hline & $\mathrm{S}$ (Ventura) & 1997 & Celery & 0 & 4 & 0 \\
\hline & $\mathrm{S}$ (Imperial) & 1998 & Broccoli & 0 & 4 & 0 \\
\hline \multirow[t]{11}{*}{ L. trifolii } & C (Monterey) & 1997 & Peppers & 8 & 0 & 0 \\
\hline & C (Monterey) & 1998 & Chrysanthemum & 0 & 8 & 0 \\
\hline & C (San Benito) & 1998 & Peppers & 5 & 0 & 0 \\
\hline & C (Tulare) & 1998 & Blackeye pea & 8 & 0 & 0 \\
\hline & S (San Diego) & 1997 & Gypsophila & 0 & 7 & 0 \\
\hline & S (Ventura) & 1997 & Celery & 0 & 7 & 1 \\
\hline & S (Ventura) & 1998 & Celery & 0 & 4 & 0 \\
\hline & S (Orange) & 1998 & Celery & 0 & 1 & 0 \\
\hline & S (Imperial) & 1998 & Lettuce & 0 & 8 & 0 \\
\hline & $\mathrm{S}$ (Imperial) & 1998 & Broccoli & 0 & 8 & 0 \\
\hline & S (San Diego) & 1998 & Celery & 0 & 8 & 0 \\
\hline
\end{tabular}

of PCR products occurred due to contamination or experimental error.

Do field populations of leafminers segregate according to host plant or geographical location? The only samples of L. trifolii collected from central California were from chrysanthemum and bell peppers and blackeye peas. These differed in their population identity; individuals collected from chrysanthemum were not significantly different from southern laboratory populations while individuals collected from bell peppers at two locations were identical to central laboratory populations.
Liriomyza trifolii individuals were collected from four host plants at six locations in southern California (Table 2). All individuals had identical marker patterns to the southern laboratory population. No L. trifolii were collected from chrysanthemum, blackeye peas, or bell peppers in southern California, so it was not possible to compare populations of L. trifolii on the same host plants but at different sites.

Liriomyza huidobrensis individuals were collected from seven host plants in central California and two host plants in southern California (Table 2). Marker patterns showed geographical separation of individuals 


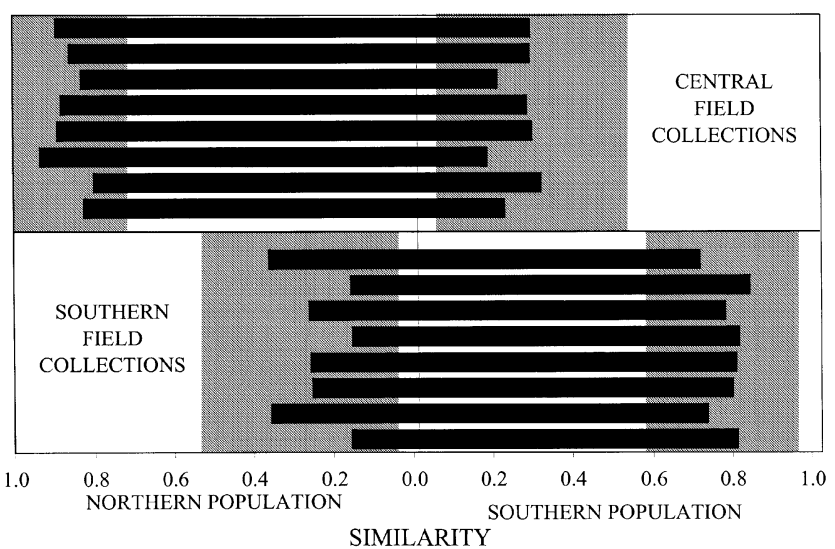

Fig. 3 Similarity of a sample of field collected individuals of L. huidobrensis from central (above) and southern (below) California when compared to central (left) and southern (right) laboratory colony marker frequencies. Shaded areas indicate $95 \%$ confidence intervals for laboratory population comparisons (see Fig. 2).

Table 3 Population identity of L. huidobrensis individuals collected from celery in Monterey and Ventura counties in 1997 and 1998

\begin{tabular}{lccccc}
\hline Collection & & & \multicolumn{2}{c}{ Identified individuals } & Unidentified \\
\cline { 1 - 1 } Location & Date & & Central & Southern & Individuals \\
\hline Monterey (C) & 1997 & & 8 & 0 & 0 \\
Monterey (C) & 1998 & & 6 & 0 & 2 \\
Ventura (S) & 1997 & & 0 & 8 & 0 \\
Ventura (S) & 1998 & & 0 & 6 & 0 \\
\hline
\end{tabular}

(Fig. 3); central field collections correlating closely to the central laboratory population and southern field collections conforming closely to the southern laboratory population $\left(F_{1,12}=206.2, P<10^{-4}\right)$. No differences were found between $L$. huidobrensis populations on host plants within each region $\left(F_{9,4}=0.637\right.$, $P=0.736$ ). Infestations of $L$. huidobrensis were found on celery and broccoli in both regions, the population identities segregated according to location but not to host plant.

Is there any evidence for population movement or mixing? The segregation of L. huidobrensis populations collected from celery host plants in Monterey (central) and Ventura (southern) counties did not differ between 1997 and 1998 (Table 3).

\section{Discussion}

Population differentiation only occurs when gene flow is interrupted between groups of individuals (Maynard
Smith, 1966). Genetic differences between leafminer populations in California may be the result of a number of mechanisms ranging from allopatric isolation at one extreme to sympatric isolation at the other. Although allopatric effects are most clearly observed with populations of organisms in their native habitat, an introduced species may display allopatry if time for population differentiation and if pressure to interrupt gene flow has been sufficient. Alternatively, allopatry may occur if introduction from two or more allopatric populations results in successful colonization of each population in different areas.

Allopatric effects appear to govern population distributions of L. huidobrensis. All individuals identified from central California share PCR fragment patterns with the central laboratory colony, while all individuals identified from southern California share patterns from the southern laboratory colony. No sympatric effects were observed for L. huidobrensis as the host plants from which insects were collected were not significant factors in governing population distributions.

The factors operating on L. trifolii population distribution are less clear. The relatively low number of samples from central California and differences in crop plants infested in each region make it impossible to ascertain whether population distribution differences are a function of host plant or geographical location. In central California, the only field-collected samples were from chrysanthemum, blackeye peas, and bell pepper. Leafminers collected from blackeye peas and bell pepper had the same identity as the central laboratory colony, but those collected from chrysanthemum shared identity with the southern laboratory colony. No L. trifolii were found on any of these host plants in southern California, even though bell pepper is cultivated widely. Furthermore, chrysanthemums are grown under glass in central California, so population differentiation of leafminers from each of the central crops may be a result of allopatric rather than sympatric effects. Leafminers on chrysanthemum may have originated from the south; the climatic and host plant differences between greenhouse and field conditions in the central region may be a greater barrier to population mixing than the geographical separation of greenhouses that share many resources.

Host plant preference and mating studies have revealed strong behavioural differences between L. trifolii laboratory populations, but not L. huidobrensis laboratory populations (Reitz et al. in prep.). Central L. trifolii populations accepted and completed development on bell peppers while southern populations could not be maintained on bell peppers. When L. trifolii from different populations were paired, mating was rare, largely due to differences in courtship behaviour. Such prezygotic differences between $L$. trifolii populations 
strongly favour host race formation and may ultimately lead to speciation. Although differences were found between $L$. huidobrensis populations, these were less marked. The results presented here support the behavioural studies; both in the genetic differences between the two populations of $L$. trifolii compared to the two populations of L. huidobrensis, and in the differences in host plants found infested at each locality in California.

The tool of PCR has allowed a number of studies to resolve and analyse insect populations (e.g. BallingerCrabtree et al., 1992; Roehrdanz et al., 1993; Haymer et al., 1997). A major difficulty in population studies using PCR technology is the difficulty of evaluating population similarities (or differences) in a statistically rigorous fashion. This stems from two constraints of standard statistical procedures; independence of variables and homoscedasticity. The problems of codependence of pairwise comparisons and the difficulty in correcting for unequal variances can be overcome by resampling procedures such as Monte Carlo, jack-knife, and bootstrap (Crowley, 1992).

Bootstrap analysis provides both a mean value and confidence intervals when evaluating sample differences. However, mean values of differences should be carefully interpreted; they are not to be mistaken for measures of genetic distances (Lynch \& Milligan, 1994). Marker studies (as opposed to true RAPD studies) will, by definition, accentuate differences so that both the mean and confidence intervals obtained are more a measure of marker efficiency than relatedness.

PCR technology has already been used to differentiate species of leafminers (Carter et al., 1993). We have shown in this study the ability to differentiate between populations of leafminers within species. PCR can be a valuable tool for identifying populations that differ in behaviour such as host plant selection, tracking population movement, and identifying origins of new infestations (Haymer \& McInnis, 1993; Garner \& Slavicek, 1996). Population identification of cosmopolitan and polyphagous pests can be invaluable in integrated pest management (Okimoto et al., 1991). Appropriate decisions regarding control methods, cultural practices, and crop choice can be made if populations that vary in pesticide resistance, host plant selection, and severity as pests are identified.

\section{References}

ARNHEIM, N., White, T. AND RAINEY, w. E. 1990. Application of PCR - organismal and population biology. Bioscience, 40, 174-182.

BALLINGER-CRABTREE, M. E., BLACK, W. C. AND MILLER, B. R. 1992. Use of genetic polymorphisms detected by the random-amplified polymorphic DNA polymerase chain reaction (RAPD-PCR) for differentiation and identification of Aedes aegypti subspecies and populations. Am. J. Trop Med. Hygiene, 47, 893-901.

CARTER, M. J., MOWAT, D. J. AND FLEMING, C. 1993. Identification of Liriomyza spp. by PCR fingerprinting. In: Ebbels, D. (ed.) British Crop Protection Council Monograph, no. 54, pp. 387-391. BCPC, Farnham, UK.

CHARLtON, C. A. AND ALlen, w. W. 1981. The biology of Liriomyza trifolii on beans and chrysanthemums. In: Schuster, D. J. (ed.) Proceedings of IFAS-Industry Conference on Biology and Control of Liriomyza Leafminers, pp. 42-49. University of Florida, Gainsville, FL.

CHIEN, C. C. AND KU, H.-C. 1996. Morphology, life history and reproduction of Liriomyza trifolii. J. Agr. Res. China, 45, 69-88.

CROWLEY, P. H. 1992. Resampling methods for computationintensive data analysis in ecology and evolution. Ann. Rev. Ecol. Syst, 23, 405-447.

GARNER, K. J. AND SLAVICEK, J. M. 1996. Identification and characterization of a RAPD-PCR marker for distinguishing Asian and North American gypsy moths. Insect Mol. Biol., 5, 81-91.

HAYMER, D. AND MCINNIS, D. 1993. Polymerase chain reaction amplification of RAPD markers to distinguish populations of the Mediterranean fruit fly, Ceratitis capitata. Heredity, 73, 302-309.

HAYMER, D. S., HE, M. AND MCINNIS, D. O. 1997. Genetic marker analysis of spatial and temporal relationships among existing populations and new infestations of the Mediterranean fruit fly (Ceratitis Capitata). Heredity, 79, 302-309.

HOY, M. А. 1994. Insect Molecular Genetics: an Introduction to Principles and Applications. Academic Press, San Diego, CA.

LANGe, W. H., GRIGARICK, A. A. AND CARlson, E. C. 1957. Serpentine leaf miner damage. Calif. Agric., 11, 3-4.

LYNCH, M. AND MilligAN, B. G. 1994. Analysis of Population Genetic Structure With Rapd Markers. Mol. Ecol., 3, 91-99.

MAYNARD SMith, J. 1966. Sympatric speciation. Am. Nat., 100, 637-650.

NEI, M. AND LI, W.-H. 1979. Mathematical models for studying genetic variation in terms of restriction endonucleases. Proc. Natl. Acad. Sci. U.S.A., 76, 5269-5273.

OKIMOTO, R., CHAMBERlin, H. M., MACFARLANE, J. L. AND wOlSTENHOLME, D. R. 1991. Repeated Sequence Sets in Mitochondrial DNA Molecules of Root Knot Nematodes (Meloidogyne) - Nucleotide Sequences, Genome Location and Potential For Host-Race Identification. Nucl. Acids Res., 19, 1619-1626.

REITZ, S. R., KUND, S., CARSON, W. G., PHILlips, P. A. AND TRUMBLE, J. T. 1999. Economics of reducing insecticide use on celery through low-input pest management strategies. Agriculture Ecosystems and Environment, 73, 185-197.

ROEHRDANZ, R. L., REED, D. K. AND BURTON, R. L. 1993. Use of Polymerase Chain Reaction and Arbitrary Primers to Distinguish Laboratory-Raised Colonies of Parasitic Hymenoptera. Biol. Control., 3, 199-206. 
SPENCER, K. A. 1973. Agromyzidae (Diptera) of Economic Importance. By: K. A. Spencer. The Hague, Junk, 1973. xi, 418 p. with illus. $24 \mathrm{~cm}$. fl 10.00. title: Series entomologica; V. 9.

VIA, S. 1984a. The quantitative genetics of polyphagy in an insect herbivore. I. Genotype-environment interaction in larval performance on different host plant species. Evolution, 38, 881-895.
VIA, S. 1984b. The quantitative genetics of polyphagy in an insect herbivore. II. Genetic correlations in larval performance within and among host plants. Evolution, 38, 896-905.

WILLIAMS, J. G. K., KUBELIK, A. R., LIVAK, K. J., RAFALSKI, J. A. AND TINGEY, S. V. 1990. DNA polymorphisms amplified by arbitrary primers are useful as genetic markers. Nucl. Acids Res., 18, 6531-6535. 